\title{
Cutting Planes from Two-Term Disjunctions
}

\author{
Pierre Bonami \\ LIF, Faculté de Sciences de Luminy, Université de Marseille, France \\ pierre.bonami@lif.univ-mrs.fr \\ Michele Conforti \\ Dipartamento di Matematica Pura e Applicata, Universitá de Padova, Italy \\ conforti@math. unipd.it \\ Gérard Cornuéjols \\ Tepper School of Business, Carnegie Mellon University, USA \\ gc0v@andrew. cmu .edu \\ Marco Molinaro \\ Tepper School of Business, Carnegie Mellon University, USA \\ molinaro@cmu.edu \\ Giacomo Zambelli \\ London School of Economics and Political Sciences, UK \\ G.Zambelli@lse.ac.uk
}

May 18, 2013

\begin{abstract}
We consider a polyhedron intersected by a two-term disjunction, and we characterize the polyhedron resulting from taking its closed convex hull. This generalizes an earlier result of Conforti, Wolsey and Zambelli on split disjunctions. We also recover as a special case the valid inequalities derived by Judice, Sherali, Ribeiro and Faustino for linear complementarity problems.
\end{abstract}

Keywords: polyhedron, two-term disjunction, convex hull, cutting plane

\section{Introduction}

We consider a polyhedron $P=\left\{x \in \mathbb{R}^{n}: A x \leq b\right\}$ and a two-term disjunction $c^{1} x \leq d_{1} \vee c^{2} x \leq$ $d_{2}$. Let $P_{1}=P \cap\left\{c^{1} x \leq d_{1}\right\}$ and $P_{2}=P \cap\left\{c^{2} x \leq d_{2}\right\}$. The main result of this paper is a description of $\overline{c o n v}\left(P_{1} \cup P_{2}\right)$, the closed convex hull of $P_{1} \cup P_{2}$. The standard disjunctive programming approach to this question is to express the closed convex hull of $P_{1} \cup P_{2}$ in a higher dimensional space and to project the resulting polyhedron onto the original $x$-space. In this paper, we follow a different approach introduced by Conforti, Wolsey and Zambelli [1].

To avoid trivial cases, we assume that $c^{1}, c^{2} \neq 0,\left(c^{1}, d_{1}\right)$ is not a multiple of $\left(c^{2}, d_{2}\right)$, and $P_{1}$ and $P_{2}$ are nonempty. Before stating our main result, we give an easy sufficient condition. 
For $w \in \mathbb{R}^{m}$, let $w^{+}$be the vector of $\mathbb{R}^{m}$ defined by $w_{i}^{+}=\max \left(0, w_{i}\right)$, and let $w^{-} \in \mathbb{R}^{m}$ be defined by $w_{i}^{-}=\max \left(0,-w_{i}\right)$.

Lemma 1. For any $\left(w, u_{0}, v_{0}\right) \in \mathbb{R}^{m+2}$ such that $w A+u_{0} c^{1}-v_{0} c^{2}=0$ and $u_{0}, v_{0} \geq 0$, the inequality $\left(w^{+} A+u_{0} c^{1}\right) x \leq \max \left\{w^{+} b+u_{0} d_{1}, w^{-} b+v_{0} d_{2}\right\}$ is valid for $\overline{\operatorname{conv}}\left(P_{1} \cup P_{2}\right)$.

Proof. Since $P_{1}$ and $P_{2}$ are nonempty, the inequality $\alpha x \leq \beta$ is valid for $\overline{\operatorname{conv}}\left(P_{1} \cup P_{2}\right)$ if and only if there exists multipliers $u, v \in \mathbb{R}^{m}, u_{0}, v_{0} \in \mathbb{R}$ such that

$$
\begin{gathered}
\alpha=u A+u_{0} c^{1} \\
\alpha=v A+v_{0} c^{2} \\
\beta \geq u b+u_{0} d_{1} \\
\beta \geq v b+v_{0} d_{2} \\
u, v, u_{0}, v_{0} \geq 0 .
\end{gathered}
$$

Given $\left(w, u_{0}, v_{0}\right)$ such that $w A+u_{0} c^{1}-v_{0} c^{2}=0$ and $u_{0}, v_{0} \geq 0$, choose $u=w^{+}, v=w^{-}$, $\alpha=w^{+} A+u_{0} c^{1}$ and $\beta=\max \left\{w^{+} b+u_{0} d_{1}, w^{-} b+v_{0} d_{2}\right\}$ and notice that this satisfies the system above.

Given a set of vectors $X \subseteq \mathbb{R}^{m}$, we say that $X$ is minimally dependent if the vectors in $X$ are linearly dependent but the vectors in every proper subset of $X$ are linearly independent.

Let $\mathcal{W}\left(A, c^{1}, c^{2}\right)$ be the set of all $\left(w, u_{0}, v_{0}\right) \in \mathbb{R}^{m+2}$ such that: (i) $u_{0}, v_{0}>0$; (ii) $w A+$ $u_{0} c^{1}-v_{0} c^{2}=0$; (iii) the set of vectors comprising $c^{1}, c^{2}$ and the rows of $A$ relative to nonzero entries of $w$ is minimally dependent. Note that $\mathcal{W}\left(A, c^{1}, c^{2}\right)$ is a finite set of vectors up to positive scaling. Indeed, finitely many row sets are considered by Condition (iii), and each of these sets gives rise to one ray.

Let $\mathcal{W}^{a}=\mathcal{W}\left(A, c^{1}, c^{2}\right)$ and $\mathcal{W}^{b}=\mathcal{W}\left((A, b),\left(c^{1}, d_{1}\right),\left(c^{2}, d_{2}\right)\right)$ where $(A, b)$ denotes the augmented matrix.

Theorem 2. The polyhedron $\overline{\operatorname{conv}}\left(P_{1} \cup P_{2}\right)$ is the set of all points in $P$ satisfying

$$
\left(w^{+} A+u_{0} c^{1}\right) x \leq \max \left\{w^{+} b+u_{0} d_{1}, w^{-} b+v_{0} d_{2}\right\} \quad \text { for every }\left(w, u_{0}, v_{0}\right) \in \mathcal{W}^{a} \cup \mathcal{W}^{b} .
$$

Theorem 2 implies the following result of Conforti, Wolsey and Zambelli [1].

Corollary 3. Consider a polyhedron $P=\left\{x \in \mathbb{R}^{n}: A x \leq b\right\}$ and a split disjunction $\pi x \leq$ $\pi_{0} \vee \pi x \geq \pi_{0}+1$ where $\pi \in \mathbb{Z}^{n}, \pi_{0} \in \mathbb{Z}$. Let $P_{1}=P \cap\left\{\pi x \leq \pi_{0}\right\}$ and $P_{2}=P \cap\left\{\pi x \geq \pi_{0}+1\right\}$. The polyhedron $\overline{\mathrm{conv}}\left(P_{1} \cup P_{2}\right)$ is the set of all points in $P$ satisfying the inequalities

$$
\pi x-s^{-} \frac{b-A x}{\lceil s b\rceil-s b} \leq \pi_{0},
$$

for all $s \in \mathbb{R}^{m}$ such that $s A=\pi, \pi_{0}<s b<\pi_{0}+1$, and the rows of $A$ relative to nonzero entries of $s$ are linearly independent.

This is the formula of the MIR inequalities of Nemhauser and Wolsey [3]. 
Proof. The case $\left(w, u_{0}, v_{0}\right) \in \mathcal{W}^{a}$ cannot occur in Theorem 2 since no valid inequality for $\overline{\operatorname{conv}}\left(P_{1} \cup P_{2}\right)$ has $w=0$ and $c^{1}=\pi$ and $c^{2}=-\pi$ are already dependent. Thus Theorem 2 reduces to: $\overline{\operatorname{conv}}\left(P_{1} \cup P_{2}\right)$ is the set of all points in $P$ satisfying the inequalities $\left(w^{+} A+u_{0} \pi\right) x \leq$ $w^{+} b+u_{0} \pi_{0}$ for every $\left(w, u_{0}, v_{0}\right) \in \mathbb{R}^{m+2}$ such that: (i) $u_{0}, v_{0}>0$; (ii) $w A+u_{0} \pi+v_{0} \pi=0$, $w b+u_{0} \pi_{0}+v_{0}\left(\pi_{0}+1\right)=0$; (iii) the set of vectors comprising $\left(\pi, \pi_{0}\right),\left(\pi, \pi_{0}+1\right)$ and the rows of $(A, b)$ relative to nonzero entries of $w$ is minimally dependent.

Since the inequality $\left(w^{+} A+u_{0} \pi\right) x \leq w^{+} b+u_{0} \pi_{0}$ is defined up to a positive scaling factor, we may assume $u_{0}+v_{0}=1$. Set $s=-w$. Thus (ii) implies $s A=\pi$ and $s b=\pi_{0}+v_{0}$. Since $u_{0}, v_{0}>0$ and $\pi_{0} \in \mathbb{Z}$, it follows that $\pi_{0}<s b<\pi_{0}+1$. Thus $\left(w^{+} A+u_{0} \pi\right) x \leq w^{+} b+u_{0} \pi_{0}$ can be rewritten as $\pi x-s^{-} \frac{b-A x}{[s b]-s b} \leq \pi_{0}$. Note that (iii) and the equation $s A=\pi$ imply that the rows of $A$ corresponding to nonzero entries of $s$ are linearly independent.

We first prove Theorem 2 for the homogeneous case where all the right-hand sides are zero.

\section{The Homogeneous Case}

We consider a polyhedral cone $P=\left\{x \in \mathbb{R}^{n}: A x \leq 0\right\}$ and a two-term disjunction $c^{1} x \leq$ $0 \vee c^{2} x \leq 0$. Let $P_{1}=P \cap\left\{c^{1} x \leq 0\right\}$ and $P_{2}=P \cap\left\{c^{2} x \leq 0\right\}$. Since both $P_{1}$ and $P_{2}$ are nonempty, an inequality $\alpha x \leq 0$ is valid for $\overline{\operatorname{conv}}\left(P_{1} \cup P_{2}\right)$ if and only if there exist multipliers $u, v \in \mathbb{R}^{m}, u_{0}, v_{0} \in \mathbb{R}$ such that

$$
\begin{gathered}
\alpha=u A+u_{0} c^{1} \\
\alpha=v A+v_{0} c^{2} \\
u, v, u_{0}, v_{0} \geq 0 .
\end{gathered}
$$

We denote by $C$ the pointed cone defined by the vectors $\left(\alpha, u, u_{0}, v, v_{0}\right)$ satisfying the above linear system. We will use the following facts.

Fact 4. Consider a point $\left(\alpha, u, u_{0}, v, v_{0}\right) \in C$. If either $u_{0}=0$ or $v_{0}=0$ then the inequality $\alpha x \leq 0$ is implied by $A x \leq 0$.

Fact 5. Let $M$ be a matrix. The rows of $M$ are minimally dependent if and only if there exists a vector $\bar{x}$ all of whose components are nonzero such that every solution of the system $x M=0$ is a multiple of $\bar{x}$.

Lemma 6. A vector $\left(\alpha, u, u_{0}, v, v_{0}\right)$ in $C$ such that $u_{0}, v_{0}>0$ is an extreme ray of $C$ if and only if $u_{i} v_{i}=0, i=1, \ldots, m$, and the following holds: the set of vectors comprising $c^{1}, c^{2}$ and the rows of $A$ corresponding to positive entries of $u+v$ is minimally dependent.

Proof. Let $\left(\alpha, u, v, u_{0}, v_{0}\right)$ be a vector in $C$ such that $u_{0}, v_{0}>0$. Then $\left(\alpha, u, v, u_{0}, v_{0}\right)$ is an extreme ray of $C$ if and only if $\left(u, v, u_{0}, v_{0}\right)$ is an extreme ray of the cone defined by

$$
\begin{aligned}
(u-v) A+u_{0} c^{1}-v_{0} c^{2} & =0 \\
u, v & \geq 0
\end{aligned}
$$


By Fact 5 , this is the case if and only if the set of rows of the matrix $\left(\begin{array}{r}A \\ -A \\ c^{1} \\ -c^{2}\end{array}\right)$ corresponding to positive entries of $\left(u, v, u_{0}, v_{0}\right)$ is minimally dependent. Since $u_{0}, v_{0}>0$, this happens if and only if $u_{i} v_{i}=0$ for all $i=1, \ldots, m$ and the set of vectors comprising $c^{1}, c^{2}$ and the rows of $A$ corresponding to positive entries of $u+v$ is minimally dependent.

Theorem 7. The cone $\overline{\operatorname{conv}}\left(P_{1} \cup P_{2}\right)$ is the set of all points in $P$ satisfying the inequalities

$$
\left(w^{+} A+u_{0} c^{1}\right) x \leq 0 \quad \text { for every }\left(w, u_{0}, v_{0}\right) \in \mathcal{W}\left(A, c^{1}, c^{2}\right) .
$$

Proof. To obtain a description of $\overline{\operatorname{conv}}\left(P_{1} \cup P_{2}\right)$ it suffices to consider inequalities $\alpha x \leq 0$ for which there exist multipliers $u, v, u_{0}, v_{0}$ such that $\left(\alpha, u, v, u_{0}, v_{0}\right)$ is an extreme ray of $C$. Furthermore, we can restrict our attention to inequalities $\alpha x \leq 0$ that are not valid for $P$. By Fact 4, this implies that $u_{0}, v_{0}>0$.

Lemma 6 implies that a vector $\left(\alpha, u, v, u_{0}, v_{0}\right) \in C$ such that $u_{0}, v_{0}>0$ is an extreme ray of $C$ if and only if $u=w^{+}$and $v=w^{-}$for some $\left(w, u_{0}, v_{0}\right) \in \mathcal{W}\left(A, c^{1}, c^{2}\right)$. Given that $\alpha=u A+u_{0} c^{1}$, the theorem follows.

\section{The Polyhedral Case}

Now we consider a polyhedron $P=\left\{x \in \mathbb{R}^{n}: A x \leq b\right\}$ and a two-term disjunction $c^{1} x \leq$ $d_{1} \vee c^{2} x \leq d_{2}$. Let $P_{1}=P \cap\left\{c^{1} x \leq d_{1}\right\}$ and $P_{2}=P \cap\left\{c^{2} x \leq d_{2}\right\}$. Our assumptions are that $c^{1}, c^{2} \neq 0,\left(c^{1}, d_{1}\right)$ is not a multiple of $\left(c^{2}, d_{2}\right)$, and $P_{1}$ and $P_{2}$ are nonempty.

Let $\tilde{P}$ be the following homogenized version of $P: \tilde{P}=\left\{\left(x, x_{0}\right) \in \mathbb{R}^{n+1}: A x-b x_{0} \leq\right.$ $\left.0, x_{0} \geq 0\right\}$. Similarly, let $\tilde{P}_{1}=\tilde{P} \cap\left\{c^{1} x-d_{1} x_{0} \leq 0\right\}$ and $\tilde{P}_{2}=\tilde{P} \cap\left\{c^{2} x-d_{2} x_{0} \leq 0\right\}$.

Lemma 8. The inequality $\alpha x \leq \beta$ is valid for $\overline{\operatorname{conv}}\left(P_{1} \cup P_{2}\right)$ if and only if the inequality $\alpha x-\beta x_{0} \leq 0$ is valid for $\overline{\operatorname{conv}}\left(\tilde{\tilde{P}}_{1} \cup \tilde{P}_{2}\right)$.

Proof. Assume $\alpha x \leq \beta$ is valid for $\overline{\operatorname{conv}}\left(P_{1} \cup P_{2}\right)$. Take $i \in\{1,2\}$; we know that $\alpha x \leq \beta$ is valid for $P_{i}$, we show that $\alpha x-\beta x_{0} \leq 0$ is valid for $\tilde{P}_{i}$. To see this, take $\left(x, x_{0}\right) \in \tilde{P}_{i}$. If $x_{0}>0$, then $\left(\frac{x}{x_{0}}, 1\right) \in P_{i}$ and thus $\alpha \frac{x}{x_{0}} \leq \beta$, or equivalently $\alpha x-\beta x_{0} \leq 0$. If $x_{0}=0$, then $\left(\begin{array}{c}A \\ c^{i}\end{array}\right) x \leq 0$ and so $x$ is in the recession cone of $P_{i}$. Validity then implies that $\alpha x \leq 0$, or equivalently $\alpha x-\beta x_{0} \leq 0$.

The converse is direct.

\section{Proof of Theorem 2.}

Let $\tilde{A}=\left(\begin{array}{cc}A & -b \\ 0 & -1\end{array}\right), \tilde{c}^{1}=\left(c^{1},-d_{1}\right)$ and $\tilde{c}^{2}=\left(c^{2},-d_{2}\right)$. From these definitions and employing Theorem 7 we have that

$$
\overline{\operatorname{conv}}\left(\tilde{P}_{1} \cup \tilde{P}_{2}\right)=\tilde{P} \cap\left\{\left(x, x_{0}\right):\left(\tilde{w}^{+} \tilde{A}+u_{0} \tilde{c}^{1}\right)\left(\begin{array}{c}
x \\
x_{0}
\end{array}\right) \leq 0 \text { for all }\left(\tilde{w}, u_{0}, v_{0}\right) \in \mathcal{W}\left(\tilde{A}, \tilde{c}^{1}, \tilde{c}^{2}\right)\right\} .
$$


Now using Lemma 8 we get that

$$
\begin{aligned}
& \overline{\operatorname{conv}}\left(P_{1} \cup P_{2}\right)=P \cap\left\{x:\left(w, w_{0}\right)^{+}\left(\begin{array}{c}
A \\
0
\end{array}\right) x+\left(w, w_{0}\right)^{+}\left(\begin{array}{l}
-b \\
-1
\end{array}\right)+u_{0} c^{1} x-u_{0} d_{1} \leq 0\right. \\
&\text { for all } \left.\left(\left(w, w_{0}\right), u_{0}, v_{0}\right) \in \mathcal{W}\left(\tilde{A}, \tilde{c}^{1}, \tilde{c}^{2}\right)\right\} \\
&=P \cap\left\{x:\left(w^{+} A+u_{0} c^{1}\right) x \leq w^{+} b+u_{0} d_{1}+w_{0}^{+}\right. \\
&\text {for all } \left.\left(\left(w, w_{0}\right), u_{0}, v_{0}\right) \in \mathcal{W}\left(\tilde{A}, \tilde{c}^{1}, \tilde{c}^{2}\right)\right\} .
\end{aligned}
$$

We claim that for $\left(\left(w, w_{0}\right), u_{0}, v_{0}\right) \in \mathcal{W}\left(\tilde{A}, \tilde{c}^{1}, \tilde{c}^{2}\right)$ we have $w^{+} b+u_{0} d_{1}+w_{0}^{+}=\max \left\{w^{+} b+\right.$ $\left.u_{0} d_{1}, w^{-} b+v_{0} d_{2}\right\}$. This is because

$$
\left(w, w_{0}\right)\left(\begin{array}{cc}
A & -b \\
0 & -1
\end{array}\right)+u_{0}\left(c^{1},-d_{1}\right)-v_{0}\left(c^{2},-d_{2}\right)=0,
$$

which implies that $w_{0}=-w b-u_{0} d_{1}+v_{0} d_{2}$; noticing that $w=w^{+}-w^{-}$, we get $w_{0}=$ $\left(w^{-} b+v_{0} d_{2}\right)-\left(w^{+} b+u_{0} d_{1}\right)$, which gives the claim.

To complete the proof of the theorem, it remains to show that if $\left(\left(w, w_{0}\right), u_{0}, v_{0}\right) \in$ $\mathcal{W}\left(\tilde{A}, \tilde{c}^{1}, \tilde{c}^{2}\right)$ then $\left(w, u_{0}, v_{0}\right) \in \mathcal{W}^{a} \cup \mathcal{W}^{b}$. If $w_{0}=0$, then it follows from definitions that $\left(w, u_{0}, v_{0}\right) \in \mathcal{W}^{b}$. Now consider the case $w_{0}>0$. Let $\left(A^{\prime}, b^{\prime}\right)$ be the submatrix of $(A, b)$ corresponding to the nonzero entries of $w$. We claim that if $N=\left(\begin{array}{cc}0 & -1 \\ A^{\prime} & -b^{\prime} \\ c^{1} & -d_{1} \\ c^{2} & -d_{2}\end{array}\right)$ is minimally dependent, then $M=\left(\begin{array}{c}A^{\prime} \\ c^{1} \\ c^{2}\end{array}\right)$ is also minimally dependent. Suppose that the rows of $M$ are not minimally dependent and let $\lambda$ be a nonzero vector with $\lambda_{j}=0$ such that $\lambda M=0$. Using the same $\lambda$, we can find a multiplier $\lambda_{0}$ for the row $(0,-1)$ such that $\left(\lambda_{0}, \lambda\right) N=0$, contradicting the fact that $N$ is minimally dependent.

\section{Complementarity Problems and Separation}

In this section, we relate Theorem 2 to previous work of Judice, Sherali, Ribeiro and Faustino [2], who proposed a valid inequality for the complementarity problem.

We first observe that our two-term disjunction problem is equivalent to the following complementarity problem: $A x=b, x \geq 0$ and $x_{1} \cdot x_{2}=0$.

We show how to transform the model $A x \leq b$ with the disjunction $c^{1} x \leq d_{1} \vee c^{2} x \leq d_{2}$ into the above form. First, unrestricted variables $x_{i}$ can be replaced by the difference of two nonnegative variables. By adding nonnegative slacks, the inequality system $A x \leq b$ can be transformed into equality form. Next consider the disjunction $c^{1} x \leq d_{1} \vee c^{2} x \leq d_{2}$. We add the two equations $c^{1} x+s_{1}^{+}-s_{1}^{-}=d_{1}$ and $c^{2} x+s_{2}^{+}-s_{2}^{-}=d_{2}$ to the current system, with $s_{1}^{+}, s_{1}^{-}, s_{2}^{+}, s_{2}^{-} \geq 0$, and we impose $s_{1}^{-} \cdot s_{2}^{-}=0$.

Conversely, in a complementarity problem $A x=b, x \geq 0, x_{1} \cdot x_{2}=0$, the complementarity condition $x_{1} \cdot x_{2}=0$ can be formulated as the disjunction $x_{1} \leq 0 \vee x_{2} \leq 0$. 
Thus Theorem 2 can be used to describe the convex hull of feasible solutions to $A x=b$, $x \geq 0, x_{1} \cdot x_{2}=0$. Multipliers satisfying (ii) in $\mathcal{W}\left(\left(\left(\begin{array}{c}A \\ -I\end{array}\right),\left(\begin{array}{l}b \\ 0\end{array}\right)\right),\left(e^{1}, 0\right),\left(e^{2}, 0\right)\right)$ give valid inequalities for $A x \leq b, x \geq 0, x_{1} \cdot x_{2}=0$ and therefore for our complementarity problem (here the unit vectors $e^{1}$ and $e^{2}$ are considered as row vectors). Condition (ii) is

$$
\begin{aligned}
& \mu A=\nu-u_{0} e^{1}+v_{0} e^{2} \\
& \mu b=0 .
\end{aligned}
$$

where $\mu$ are the multipliers associated with the constraint $A x \leq b$ and $\nu$ the multipliers associated with $-x \leq 0$.

Using $A x=b$, cut (1) becomes

$$
\sum_{j} \nu_{j}^{+} x_{j}-u_{0} x_{1} \geq 0
$$

Assume that we have a tableau for problem $A x=b, x \geq 0$ where $x_{1}$ and $x_{2}$ are both basic and strictly positive:

$$
\begin{aligned}
& x_{1}+\sum_{j \in N} \bar{a}_{1 j} x_{j}=\bar{b}_{1} \\
& x_{2}+\sum_{j \in N} \bar{a}_{2 j} x_{j}=\bar{b}_{2} .
\end{aligned}
$$

We can use formula (6) to cut off the current solution $\bar{x}$ which is infeasible since $\bar{x}_{1} \cdot \bar{x}_{2}=$ $\bar{b}_{1} \cdot \bar{b}_{2}>0$. Multiplying (7) by $1 / \bar{b}_{1}$ and $(8)$ by $1 / \bar{b}_{2}$ and subtracting we get

$$
-\frac{x_{1}}{\bar{b}_{1}}+\frac{x_{2}}{\bar{b}_{2}}+\sum_{j \in N}\left(\frac{\bar{a}_{2 j}}{\bar{b}_{2}}-\frac{\bar{a}_{1 j}}{\bar{b}_{1}}\right) x_{j}=0 .
$$

This equality is obtained from the system $A x=b$ using multipliers $\mu$ such that $\mu b=0$. Let $\nu, u_{0}, v_{0}$ satisfy (4). Since $\mu A$ are the coefficients in the left-hand-side of (9), this implies $u_{0}=1 / \bar{b}_{1}, v_{0}=1 / \bar{b}_{2}$, and $\nu_{j}=\left(\frac{\bar{a}_{2 j}}{b_{2}}-\frac{\bar{a}_{1 j}}{b_{1}}\right)$ for all $j \in N$. As observed above, these multipliers will produce a valid inequality using formula (6). The corresponding cut is

$$
-\frac{x_{1}}{\bar{b}_{1}}+\sum_{j \in N}\left(\frac{\bar{a}_{2 j}}{\bar{b}_{2}}-\frac{\bar{a}_{1 j}}{\bar{b}_{1}}\right)^{+} x_{j} \geq 0 .
$$

This is the cut of Judice, Sherali, Ribeiro and Faustino [2].

Acknowledgement: This work was supported in part by NSF grant CMMI 1263239 and ONR grant N00014-12-10032. 


\section{References}

[1] Michele Conforti, Laurence Wolsey and Giacomo Zambelli, Split, MIR and Gomory inequalities (July 2012).

[2] Joaquim J. Júdice, Hanif Sherali, Isabel M. Ribeiro and Ana M. Faustino, A complementarity-based partitioning and disjunctive cut algorithm for mathematical programming problems with equilibrium constraints, Journal of Global Optimization 136 (2006) 89-114.

[3] George L. Nemhauser and Laurence A. Wolsey, A recursive procedure to generate all cuts for 0-1 mixed integer programs, Mathematical Programming 46 (1990) 379-390. 